\section{Prevalência de transtornos mentais comuns em populações atendidas pelo Programa Saúde da Família (QUALIS) no Município de São Paulo, Brasil}

\author{
Prevalence of common mental disorders \\ in a population covered by the Family Health \\ Program (QUALIS) in São Paulo, Brazil
}

Luciana Maragno 1

Moisés Goldbaum 1

Reinaldo José Gianini 1

Hillegonda Maria Dutilh Novaes 1

Chester Luiz Galvão César 2

\footnotetext{
1 Faculdade de Medicina Universidade de São Paulo, São Paulo, Brasil.

2 Faculdade de Saúde Pública, Universidade de

São Paulo, São Paulo, Brasil.

Correspondência R. J. Gianini

Departamento de Medicina Preventiva Faculdade de Medicina, Universidade de São Paulo. Av. Dr. Arnaldo 455, o andar, São Paulo, SP 01246-903, Brasil. reinaldo@sorocaba.pucsp.br
}

\begin{abstract}
The prevalence of common mental disorders has increased in many countries. Cases are often not identified and adequately treated because traditional health care services are rarely prepared to deal with this problem. The Family Health Program (FHP) has been implemented in Brazil since 1995-1996 and provides a new primary health care model with the potential for better care for these patients. This study investigates common mental disorders prevalence according to FHP coverage and associated socio-demographic factors. A large health and health care survey was conducted from January to March 2001 in areas partly covered by the FHP in a peripheral area of the city of Sao Paulo and included common mental disorders screening in 2,337 individuals $>15$ years of age. There was no significant difference in common mental disorders prevalence according to FHP. Common mental disorders prevalence was significantly higher among females $(P R=1.34)$, elderly $(P R=$ 1.56), and individuals with lower income ( $P R=$ $2.64)$ or less schooling $(P R=2.83)$. Common mental disorders was associated with indicators of social disadvantage, implying the need to focus on specific health problems and risk groups to improve the impact of care.
\end{abstract}

Mental Disorders; Family Health Program; Services Evaluation

\section{Introdução}

Estudos epidemiológicos mostram que milhões de pessoas sofrem algum tipo de doença mental no mundo e que este número vem sofrendo um aumento progressivo, principalmente nos países em desenvolvimento ${ }^{1}$. Casos com sintomas ansiosos, depressivos ou somatoformes, mesmo não satisfazendo todos os critérios diagnósticos de doença mental, apresentam uma elevada prevalência na população adulta ${ }^{2}$. Entretanto, apenas uma pequena parte deles é identificada e tratada $3,4,5,6$, aumentando o sofrimento individual e com implicações socioeconômicas significativas, pois tais sintomas constituem causa importante de dias perdidos de trabalho, além de elevarem a demanda nos serviços de saúde 7 .

Segundo Santos 6, transtorno mental comum (TMC) se refere à situação de saúde de uma população com indivíduos que não preenchem os critérios formais para diagnósticos de depressão e/ou ansiedade segundo as classificações DSM-IV (Diagnostic and Statistical Manual of Mental Disorders - Fourth Edition) e CID-10 (Classificação Internacional de Doenças - 10a Revisão), mas que apresentam sintomas proeminentes que trazem uma incapacitação funcional comparável ou até pior do que quadros crônicos já bem estabelecidos. Ainda, segundo Santos 6, esse quadro clínico, em geral, não faz os pacientes procurarem a assistên- 
cia necessária, e muitas vezes, quando procuram esta assistência, são subdiagnosticados, podendo, desta forma, não receber o tratamento adequado. No campo da atenção primária, ou da prática médica não-psiquiátrica, isto se torna ainda mais relevante se levarmos em consideração a presença de comorbidades, que acabam por agravar o prognóstico de ambos os problemas, tanto por piora do quadro clínico principal, quanto por aderência inadequada aos tratamentos propostos 6 .

Anteriormente as estimativas de prevalência de problemas mentais eram obtidas a partir de populações institucionalizadas, sendo que os estudos comunitários só começaram a ser desenvolvidos após a Segunda Guerra Mundial. As pesquisas epidemiológicas primariamente desenvolvidas em ambientes asilar e hospitalar passaram a incluir os serviços de saúde ambulatoriais e, posteriormente, se estenderam para estudos de base populacional. Essa mudança na população estudada e o desenvolvimento da epidemiologia psiquiátrica tornaram possível conhecer melhor a morbidade, em que cerca de $90 \%$ das manifestações psiquiátricas compõem-se de distúrbios não-psicóticos, principalmente depressão e ansiedade, incluindo sintomas como insônia, fadiga, irritabilidade, dificuldade de memória e de concentração e queixas somáticas 2 .

No Brasil, ainda é muito pequeno o número de investigações epidemiológicas de base populacional, especialmente na área de saúde mental. Entretanto, nas últimas décadas, isto vem se modificando 8 . Em Porto Alegre e São Paulo, cerca de $50 \%$ dos pacientes que procuram os serviços primários de saúde são considerados portadores de distúrbios mentais não-psicóticos 2 . Em Pelotas a prevalência de transtornos psiquiátricos menores foi de $22,7 \%$, sendo $17,9 \%$ entre os homens e $26,5 \%$ entre as mulheres 8 . No Rio de Janeiro, em 1995, observou-se a presença desses quadros em cerca de um terço dos pacientes de um ambulatório geral universitário 2 . Em Recife, um estudo populacional 7 encontrou uma prevalência total de transtornos mentais comuns de, aproximadamente, $35 \%$.

Comparando-se esses dados com alguns estudos feitos em outros países, tem-se que a prevalência desses transtornos varia de $23,9 \%$ em comunidade rural na África do Sul 9 a 52\% em Santiago, Chile 10. Verifica-se, portanto, que os nossos padrões epidemiológicos são semelhantes.

Os estudos sobre saúde mental têm verificado associação dos TMC com algumas variáveis sócio-demográficas. Quanto ao sexo, os estudos descrevem prevalência maior nas mu- lheres 2,8. Com relação à idade, os estudos apresentam resultados diversos: Lima et al. 8 referem aumento linear da prevalência de acordo com a idade; entretanto, os estudos de Coutinho et al. 2 e Andrade et al. 5 descrevem prevalência menor nos maiores de 44 anos e maiores de 60 anos, respectivamente. Quanto às condições sócio-econômicas, escolaridade e renda, aceita-se que existe uma relação inversamente proporcional entre estas variáveis e a prevalência de TMC. Quanto à procedência, observouse, em motoristas e cobradores de ônibus residentes no Município de São Paulo, que aqueles procedentes, migrantes, da Região Nordeste, apresentavam prevalência maior quando comparados aos de outras regiões 11. Em relação à situação conjugal, os estudos indicam menor prevalência em indivíduos solteiros, quando comparados aos casados. Porém, esta associação é fraca e não se manteve significante quando os dados foram ajustados segundo as categorias de escolaridade no estudo de Coutinho et al. 2 .

A realização de estudos de base populacional sobre as condições de vida e saúde (e entre eles estudos sobre TMC), nas áreas onde ocorre a implantação do Programa Saúde da Família (PSF), pode fornecer subsídios que orientem suas ações e avaliem seu desenvolvimento. O PSF no Município de São Paulo teve seu início com o Programa QUALIS, em Itaquera, em 1996, e Parque São Lucas, Sapopemba e Vila Nova Cachoeirinha, em 1997. O PSF tem se constituído em uma estratégia de reorganização do sistema de atenção à saúde e tem sido descrito como uma ferramenta de eqüidade na oferta de serviços 12,13. Tendo se difundido em âmbito nacional a partir de 1995/1996, se propõe a realizar atenção primária com prioridade para as populações de risco, incluindo não só critérios biológicos, mas também sócio-econômicos. O PSF opera com uma abordagem da família, em integração com a comunidade, fazendo a busca ativa de casos com intervenção oportuna e precoce, dando ênfase à prevenção e educação em saúde. Propugna por extensão de cobertura e facilitação do acesso, continuidade das ações de saúde, trabalho de equipe multiprofissional e elevada resolutividade 14,15. Nesse sentido, teria potencial para melhor atuação sobre problemas como os TMC, do que a atenção primária tradicional.

O presente estudo objetiva investigar a prevalência de TMC segundo a cobertura PSFQUALIS e analisar a sua distribuição segundo determinadas variáveis sócio-demográficas. 


\section{Métodos}

\section{Local e delineamento do estudo}

Este subprojeto faz parte de um projeto intitulado Estudo de Morbidade e de Utilização de Serviços de Saúde na População Coberta pelo Projeto QUALIS, componente do projeto Novos Modelos de Assistência à Saúde: Avaliação do Programa de Saúde da Família (PSF) no Município de São Paulo.

É um estudo transversal, cuja coleta de dados foi conduzida no período de janeiro a março de 2001, em dois distritos atendidos pelo Projeto QUALIS - Vila Nova Cachoeirinha e Vila Curuçá, no Município de São Paulo. Foi analisado e aprovado pelo Comitê de Ética do Hospital das Clínicas, Faculdade de Medicina, Universidade de São Paulo (CAPPesq).

\section{População do estudo}

A população analisada refere-se aos maiores de 15 anos residentes nos Distritos de Vila Curuçá e Vila Nova Cachoeirinha, Município de São Paulo, divididos em quatro subgrupos:

- População residente no Distrito de Vila Nova Cachoeirinha, dentro da área de abrangência do Projeto QUALIS, unidades de Vila Galvão e Ilza Hutzler (estimada em 23.277 indivíduos maiores de 15 anos);

- População residente no Distrito de Vila Nova Cachoeirinha, fora da área de abrangência do Projeto QUALIS (estimada em 46.742 indivíduos maiores de 15 anos);

- População residente no Distrito de Vila Curuçá, dentro da área de abrangência do Projeto QUALIS, unidades de Silva Teles e Santa Rita (estimada em 15.583 indivíduos maiores de 15 anos);

- População residente no Distrito de Vila Curuçá, fora da área de abrangência do Projeto QUALIS (estimada em 28.147 indivíduos maiores de 15 anos).

\section{Instrumentos}

Utilizou-se como instrumento de pesquisa sobre saúde mental o Self Report Questionnaire (SRQ-20), derivado de quatro outros instrumentos já existentes e desenvolvido por Harding 16. O SRQ-20 foi recomendado pela Organização Mundial da Saúde (OMS) para estudos comunitários e em atenção primária à saúde. Este instrumento que se compõe originalmente de 24 questões de fácil resposta (tipo sim/não), sendo as vinte primeiras objeto deste trabalho, para detecção de possíveis portadores (suspei- tos) de distúrbios neuróticos e, as outras quatro para distúrbios psicóticos. O SRQ-20 foi validado para a população brasileira por Mari \& Willians 16, sendo observadas sensibilidade de $83 \%$ e especificidade de $80 \%$; e o ponto de corte utilizado foi de 5-6 para os homens e de 7-8 para as mulheres, que representaram os melhores pontos de sensibilidade e especificidade do instrumento.

Além de informações sobre saúde mental obtidas na aplicação do SRQ-20 no inquérito, pesquisou-se informações sobre dados referentes às seguintes características sociodemográficas: idade, sexo, estado civil, escolaridade (número total de anos completados na escola), procedência (natural do Estado de São Paulo ou de outros Estados) e renda per capita mensal (calculada como o número de salários mínimos - correspondendo ao valor deste na época de coleta de dados, $\mathrm{R} \$ 151,00$ - ganhos por família dividido pelo número de pessoas que moravam na casa avaliada).

Seleção e tamanho da amostra, unidade de análise e critérios de seleção

Para cada subgrupo foram adotados como unidades primárias e secundárias de amostragem, respectivamente, o setor censitário e o domicílio. Todos os moradores dos domicílios sorteados foram incluídos na amostra e entrevistados. Para os subgrupos fora da área de abrangência do PSF foram sorteados trinta setores censitários, com probabilidade proporcional ao tamanho do distrito, e dentro deles foram sorteados dez domicílios. As áreas de abrangência do PSF foram divididas em microareas, que corresponderam aos espaços geográficos cobertos por um único agente de saúde. Para cada microarea (de um total de trinta) foram sorteados dez domicílios. Esse procedimento permitiu identificar em cada subgrupo trezentas famílias.

\section{Coleta dos dados}

Os questionários foram aplicados por entrevistadores durante visita domiciliar. O SRQ-20 foi aplicado a todos os indivíduos maiores de 15 anos dos domicílios amostrados. As informações sobre renda per capita familiar foram complementadas por questões dirigidas aos chefes de família.

\section{Análise dos dados}

Os dados, após codificação, foram digitados em um banco de dados utilizando-se o programa Epi Info 6.0 (Centers for Disese Control and 
Prevention, Atlanta, Estados Unidos). O banco de dados original foi transferido para o programa Stata 7.0 (Stata Corporation, College Station, Estados Unidos), para realização da análise por conglomerados. Foi criada uma variável TMC a partir do escore total de cada indivíduo no SRQ-20, no qual os sujeitos foram classificados como portador de TMC ou não, de acordo com os pontos de corte, já especificados anteriormente. Foi realizada análise descritiva da população estudada segundo as variáveis em questão (TMC, idade, sexo, estado civil, escolaridade, procedência, renda mensal e subgrupos especificados anteriormente). Posteriormente, foi realizada a análise univariada para examinar associações de variáveis explanatórias com o risco de apresentar TMC para o conjunto total das populações estudadas. Foram estimadas razões de prevalência com cálculo de intervalos de $95 \%$ de confiança. Adotou-se um nível de significância igual a 0,05 para julgar as associações. Empregou-se a estimativa de proporções própria para conglomerados e o teste do qui-quadrado de Pearson, corrigido pela aproximação de Satterwaite e transformado em distribuição F, segundo sugestão de Rao \& Thomas. A análise multivariada foi utilizada para estimar a contribuição independente de cada variável para a chance de apresentar TMC. Para a construção do modelo foram testadas todas as variáveis que na análise univariada apresentaram associação significante com TMC. Para tanto, empregou-se a regressão de Poisson para conglomerados, utilizando-se o programa Stata 7.0.

\section{Resultados}

Uma primeira análise, descrita na Tabela 1, mostra a comparação das variáveis sociodemográficas e prevalência de TMC entre dois grupos populacionais (cobertos e não cobertos pelo PSF). Observa-se que não há diferença estatisticamente significante na prevalência de TMC segundo a cobertura PSF. Entretanto, existe diferença estatisticamente significante na distribuição proporcional segundo a idade, localizada no grupo com 65 anos ou mais. Quanto à escolaridade e renda, observa-se na área não coberta uma maior concentração, estatisticamente significante, de indivíduos com escolaridade superior e renda per capita familiar de cinco salários mínimos ou mais. Não se observam diferenças quanto ao sexo, estado civil e procedência.

Diante dessas evidências, ou seja, a ausência de diferenças de prevalência de TMC entre as duas populações, optou-se por realizar uma análise de TMC segundo os fatores estudados agrupando-se as mesmas. No conjunto da população analisada, a estimativa de prevalência de TMC é de 24,95\% (IC95\%: 22,04-28,10).

Na Tabela 2 nota-se que as variáveis sociodemográficas avaliadas (sexo, idade, estado civil, escolaridade, procedência e renda per capita) se mostraram associadas aos TMC. Com relação ao sexo, a prevalência de TMC foi significantemente maior no sexo feminino. Quanto à idade, observou-se tendência linear $(p<0,001)$, com aumento significante na prevalência de TMC a partir dos 45 anos, sendo que a maior razão de prevalência (RP) encontrada foi na faixa etária de 65 anos ou mais. Analisando os resultados obtidos em relação ao estado civil, tomando como referência a categoria dos solteiros, encontrou-se uma prevalência dos transtornos estudados maior nas categorias "divorciados ou separados, judicialmente ou não" e "viúvos". Observou-se uma tendência linear de aumento da prevalência de TMC em função do menor grau de escolaridade. Com relação à procedência, verificou-se uma maior prevalência de TMC entre os indivíduos procedentes de outros estados. E, quanto à renda per capita, as categorias de menor renda apresentaram prevalência de TMC significantemente maiores quando comparadas à categoria de maior renda (tendência linear com $\mathrm{p}<0,001$ ).

Na Tabela 3 são descritos os resultados da análise multivariada, realizada com o objetivo de atenuar o possível efeito confundimento existente entre as variáveis estudadas, modelo no qual se incluiu inicialmente todas as variáveis sócio-demográficas que se apresentaram associadas aos TMC. Observou-se, entretanto, que na primeira etapa desta análise a associação da variável procedência com TMC perdeu significância estatística quando ajustada por escolaridade, sendo, portanto, desconsiderada nas análises subseqüentes e no modelo final.

Nos resultados finais obtidos neste modelo, observa-se que a prevalência de TMC se mantém maior no sexo feminino e nos indivíduos entre 45 e 54 anos. Para estado civil, o modelo mostra que os casados, em relação aos solteiros, passam a apresentar menor prevalência; porém as diferenças na prevalência encontradas na análise univariada entre os divorciados ou separados, e entre os viúvos, não se confirmam na análise multivariada. Com relação à associação TMC e escolaridade, continua-se a observar uma queda nos valores da razão de prevalência de TMC em direção a categorias de maior escolaridade, que são estatisticamente significantes, de forma a configurar uma ten- 
Descrição das amostras segundo variáveis sócio-demográficas e transtornos mentais comuns. Vila Curuçá e Vila Nova Cachoeirinha, São Paulo, Brasil, 2001.

\begin{tabular}{|c|c|c|c|c|c|c|c|}
\hline \multirow[t]{2}{*}{ Variável } & \multicolumn{3}{|c|}{ Área coberta pelo PSF } & \multicolumn{3}{|c|}{ Área não coberta pelo PSF } & \multirow[t]{2}{*}{$\mathbf{P}$} \\
\hline & $N$ & $\%$ & IC95\% & $N$ & $\%$ & IC95\% & \\
\hline Sexo & & & & & & & 0,7699 \\
\hline Masculino & 530 & 43,88 & $41,9-45,9$ & 469 & 43,39 & $40,8-46,0$ & \\
\hline Feminino & 681 & 56,12 & $54,1-58,1$ & 657 & 56,61 & $54,0-59,2$ & \\
\hline Idade (anos) & & & & & & & 0,0108 \\
\hline $15-24$ & 310 & 25,44 & $23,0-28,1$ & 319 & 24,31 & $20,3-28,9$ & \\
\hline $25-34$ & 243 & 19,51 & $17,0-22,3$ & 263 & 26,36 & $22,0-31,2$ & \\
\hline $35-44$ & 240 & 19,6 & $17,3-22,1$ & 236 & 20,49 & $17,8-23,5$ & \\
\hline $45-54$ & 186 & 15,8 & $13,7-18,1$ & 142 & 12,97 & $10,7-15,6$ & \\
\hline $55-64$ & 117 & 9,73 & $7,9-11,9$ & 98 & 9,84 & $7,6-12,6$ & \\
\hline 65 ou mais & 115 & 9,93 & $8,1-12,2$ & 68 & 6,02 & $4,5-8,0$ & \\
\hline Estado civil & & & & & & & 0,1925 \\
\hline Solteiro & 430 & 35,81 & $32,7-39,0$ & 396 & 33,12 & $29,5-36,9$ & \\
\hline Casado & 460 & 37,83 & $34,2-41,6$ & 426 & 41,27 & $37,5-45,2$ & \\
\hline $\begin{array}{l}\text { Divorciado ou separado, } \\
\text { judicialmente ou não }\end{array}$ & 64 & 5,21 & $4,0-6,7$ & 56 & 4,62 & $3,3-6,4$ & \\
\hline "Concubinato" & 164 & 13,38 & $10,5-16,9$ & 183 & 15,82 & $11,9-20,8$ & \\
\hline Viúvo & 93 & 7,77 & $6,4-9,4$ & 65 & 5,17 & $3,7-7,2$ & \\
\hline Escolaridade & & & & & & & 0,0168 \\
\hline Analfabeto & 69 & 5,67 & $4,2-7,6$ & 53 & 4,3 & $3,2-5,7$ & \\
\hline 1a a 4a série do ensino fundamental & 334 & 27,73 & $25,2-30,5$ & 297 & 23,86 & $20,7-27,3$ & \\
\hline 5a a $8 \underline{a}$ série do ensino fundamental & 377 & 30,70 & $28,0-33,6$ & 338 & 30,12 & $25,7-35,0$ & \\
\hline 1a a $3 \underline{a}$ série do ensino médio & 365 & 30,42 & $27,5-33,6$ & 344 & 31,75 & $28,5-35,2$ & \\
\hline $\begin{array}{l}\text { Curso superior incompleto } \\
\text { ou completo }\end{array}$ & 65 & 5,48 & $4,1-7,6$ & 93 & 9,97 & $7,6-13,0$ & \\
\hline Procedência & & & & & & & 0,7668 \\
\hline Estado de São Paulo & 720 & 60,64 & $57,2-64,0$ & 671 & 61,52 & $56,6-66,2$ & \\
\hline Outros & 491 & 39,36 & $36,0-42,8$ & 455 & 38,48 & $33,8-43,4$ & \\
\hline Renda per capita (salários mínimos) & & & & & & & 0,0439 \\
\hline$<0,50$ & 132 & 11,12 & $8,1-15,0$ & 144 & 11,42 & $7,9-16,2$ & \\
\hline $0,50-0,99$ & 231 & 18,9 & $15,3-23,1$ & 193 & 15,11 & $11,2-20,1$ & \\
\hline $1,00-1,99$ & 361 & 30,34 & $25,4-35,7$ & 309 & 26,94 & $21,9-32,6$ & \\
\hline $2,00-4,99$ & 384 & 34,54 & $28,9-40,6$ & 316 & 33,82 & $27,6-40,6$ & \\
\hline$\geq 5,00$ & 57 & 5,11 & $3,3-7,7$ & 107 & 12,71 & $8,8-18,0$ & \\
\hline Transtorno mental comum & & & & & & & 0,3526 \\
\hline Sim & 326 & 24,1 & $20,1-28,6$ & 308 & 26,6 & $23,8-29,6$ & \\
\hline Não & 885 & 75,9 & $71,4-79,9$ & 818 & 73,4 & $70,4-76,2$ & \\
\hline Total & 1.211 & 100,0 & & 1.126 & 100,0 & & \\
\hline
\end{tabular}


Tabela 2

Transtornos mentais comuns (TMC) segundo fatores sociais e demográficos.

Vila Curuçá e Vila Nova Cachoeirinha, São Paulo, Brasil, 2001.

\begin{tabular}{|c|c|c|c|}
\hline Variável & TMC (\%) & RP (IC95\%) & Valor de $p$ \\
\hline Sexo & & & 0,0029 \\
\hline Masculino & 20,94 & 1,00 & \\
\hline Feminino & 27,99 & $1,34(1,10-1,62)$ & \\
\hline Idade $^{*}$ (anos) & & & 0,0011 \\
\hline $15-24$ & 21,55 & 1,00 & \\
\hline $25-34$ & 20,77 & $0,96(0,71-1,30)$ & \\
\hline $35-44$ & 22,38 & $1,03(0,80-1,33)$ & \\
\hline $45-54$ & 32,77 & $1,53(1,15-2,04)$ & \\
\hline $55-64$ & 31,12 & $1,46(1,04-2,05)$ & \\
\hline 65 ou mais & 33,66 & $1,56(1,11-2,21)$ & \\
\hline Estado civil & & & $<0,001$ \\
\hline Solteiro & 23,24 & 1,00 & \\
\hline Casado & 21,39 & $0,93(0,77-1,12)$ & \\
\hline $\begin{array}{l}\text { Divorciado ou separado, } \\
\text { judicialmente ou não }\end{array}$ & 38,13 & $1,65(1,25-2,17)$ & \\
\hline "Concubinato" & 27,15 & $1,18(0,91-1,53)$ & \\
\hline Viúvo & 41,67 & $1,81(1,33-2,47)$ & \\
\hline Escolaridade $^{*}$ & & & $<0,001$ \\
\hline Analfabeto & 38,81 & $2,83(1,74-4,61)$ & \\
\hline 1a a $4 \underline{a}$ série do ensino fundamental & 32,54 & $2,38(1,50-3,77)$ & \\
\hline 5ạ a 8a série do ensino fundamental & 26,56 & $1,95(1,23-3,10)$ & \\
\hline 1ạ a 3a série do ensino médio & 17,98 & $1,30(0,83-2,03)$ & \\
\hline Superior incompleto ou completo & 13,78 & 1,00 & \\
\hline Procedência & & & 0,0042 \\
\hline Estado de São Paulo & 22,28 & 1,00 & \\
\hline Outros & 29,08 & $1,31(1,09-1,57)$ & \\
\hline $\begin{array}{l}\text { Renda per capita } \\
\text { (salários mínimos)* }^{\text {(sam }}\end{array}$ & & & $<0,001$ \\
\hline$<0,5$ & 30,10 & $2,64(1,33-5,23)$ & \\
\hline $0,50-0,99$ & 31,24 & $2,74(1,45-5,18)$ & \\
\hline $1,00-1,99$ & 28,37 & $2,49(1,30-4,77)$ & \\
\hline $2,00-4,99$ & 20,49 & $1,80(0,97-3,34)$ & \\
\hline$\geq 5,0$ & 11,39 & 1,00 & \\
\hline
\end{tabular}

* Valor de p na tendência linear menor que 0,001.

dência linear $(\mathrm{p}<0,01)$. Sobre a variável renda per capita, observa-se RP crescentes quanto menor a renda, também com tendência linear significante $(\mathrm{p}<0,01)$. Finalmente, segundo a cobertura PSF, confirma-se, na análise multivariada, a ausência de diferença de prevalência de TMC, salientando-se que no modelo final observa-se uma evidente diminuição da RP (de 0,91 para 0,99 ).

\section{Discussão}

O grande número de participantes e a perda mínima de informação são dois aspectos muito positivos neste estudo. Trata-se, contudo, de uma amostra da periferia do Município de São Paulo que apresenta, quando comparada com todo o município, uma menor variabilidade em suas condições sócio-econômicas, o que poderia limitar certos achados, como por exemplo, associações significantes ou efeitos de maior magnitude, decorrentes do viés de seleção. Quanto ao viés de confundimento, tentou-se controlá-lo por meio da análise multivariada dos dados, mas, ainda assim, pode ser que persista algum grau deste vício devido às complexas relações entre as variáveis estudadas. Quanto ao desenho, por se tratar de estudo transversal, não é possível estudar a causalidade reversa das relações analisadas entre TMC e condições sócio-econômicas. Deve-se levar em conta que o SRQ-20 simplesmente rastreia casos suspeitos de TMC, sendo o padrão-ouro para diagnóstico a entrevista com psiquiatra 17 . Neste estudo podemos avaliar seu Valor Preditivo Positivo em 59\% e seu Valor Preditivo Negativo em $94 \%$, considerando-se sensibilidade de 0,85, especificidade de 0,80 17 e prevalência de $25 \%$. Assim, há um viés de informação relevante a se considerar que pode atenuar a associação entre TMC e as variáveis pesquisadas.

O principal achado deste trabalho é ter encontrado tanto nas áreas cobertas quanto nas áreas não cobertas pelo PSF, uma associação significante entre a ocorrência de casos suspeitos de TMC e os indicadores de desvantagem social. Esses achados não diferem substancialmente daquilo que tem sido descrito na literatura a respeito da relação entre variáveis sóciodemográficas e TMC 2,9. A prevalência de casos suspeitos de TMC no conjunto da população analisada, $24,95 \%$, é consistente com os resultados encontrados em outros estudos nacionais 7,8 e mostra-se associada ao sexo feminino, aos indivíduos de maior idade, à baixa escolaridade e à menor renda per capita $3,10,18,19$.

A determinação social seria uma hipótese explicativa para esses resultados. Derivada da teoria dos eventos de vida e da teoria dos papéis sociais, defende que determinados acontecimentos na vida dos indivíduos, denominados eventos de vida produtores de estresse 20 , atuariam como estressores não-específicos ao provocarem alterações no sistema endócrino, aumentando o risco de desenvolvimento de doenças, dentre as quais os distúrbios mentais, e considera, ainda, a identidade social dos indivíduos (e seus respectivos desajustes) como 
um conjunto de comportamentos associados à posição que ocupam na rede social 2. Vários autores ao analisar tais relações, associam-nas à questão do poder, ou então, ao desequilíbrio entre dominação e subordinação econômica, política ou social 7,18,21. Dentro deste enfoque é possível compreender os resultados obtidos.

A maior prevalência de TMC entre as mulheres pode ser atribuída à dinâmica dos gêneros nas relações de poder, que pode resultar em opressão para esta categoria 10 . Um aspecto que se faz digno de nota em relação à RP de TMC, segundo o sexo, é que o valor obtido foi menor que o encontrado na literatura $3,8,10$. Tal achado pode ser explicado pelo fato da análise ter sido feita em uma amostra na qual as demais variáveis sócio-demográficas, especialmente a renda per capita, apresentavam relativamente menor variabilidade, o que pode ter atenuado a associação.

Em relação à associação TMC e indivíduos de faixa etária mais elevada, algumas das justificativas seriam maior vulnerabilidade deste grupo etário no que se refere a distúrbios psiquiátricos, acúmulo de doenças crônicas preexistentes, dificuldades econômicas, isolamento social, desmerecimento social e a maior ocorrência de eventos de vida produtores de estresse 2,10,21.

Não há consenso na literatura quanto à associação TMC e estado civil. Este trabalho oferece um novo dado de conhecimento, ou seja, uma menor prevalência de TMC entre os indivíduos casados nesta população quando os resultados são ajustados por sexo, idade, escolaridade e renda. Esse efeito independente da variável estado civil relativo à categoria "casados" difere de outros estudos 2,3. Explicações plausíveis para esse dado podem ser feitas levandose em consideração a mesma hipótese citada acima (de determinação social), supondo-se que os indivíduos casados dispõem de maior suporte (familiar/social) 17. Porém é importante que se faça a ressalva correspondente à possibilidade de causalidade reversa (indivíduos com TMC podem ter menor chance de casar), cujas características deste estudo (estudo transversal) impedem a análise.

Quanto à escolaridade, sabe-se que esta aumenta a possibilidade de escolhas na vida, além de influenciar aspirações, auto-estima e aquisição de novos conhecimentos, que podem motivar atitudes e comportamentos mais saudáveis. Desta maneira, influencia as condições sócio-econômicas futuras e sua inserção na estrutura ocupacional, logo, seu papel social. Levando-se em conta tais considerações, a sua falta diminui o poder de decisão do indivíduo, gerando uma relativa incapacidade de influen-
Tabela 3

Análise multivariada da associação entre TMC e fatores sócio-demográficos.

Vila Curuçá e Vila Nova Cachoeirinha, São Paulo, Brasil, 2001*.

\begin{tabular}{|c|c|c|c|}
\hline Variável & RP & IC95\% & Valor de $p$ \\
\hline \multicolumn{4}{|l|}{ Sexo } \\
\hline Masculino & 1,00 & & \\
\hline Feminino & 1,28 & $1,07-1,53$ & 0,008 \\
\hline \multicolumn{4}{|l|}{ Idade (anos) } \\
\hline $15-24$ & 1,00 & & \\
\hline $25-34$ & 1,07 & $0,79-1,43$ & 0,669 \\
\hline $35-44$ & 1,15 & $0,88-1,51$ & 0,302 \\
\hline $45-54$ & 1,62 & $1,10-2,39$ & 0,016 \\
\hline $55-64$ & 1,48 & $0,97-2,26$ & 0,068 \\
\hline 65 ou mais & 1,35 & $0,88-2,06$ & 0,170 \\
\hline \multicolumn{4}{|l|}{ Estado civil } \\
\hline Solteiro & 1,00 & & \\
\hline Casado & 0,71 & $0,56-0,91$ & 0,008 \\
\hline $\begin{array}{l}\text { Divorciado ou separado, } \\
\text { judicialmente ou não }\end{array}$ & 0,99 & $0,70-1,40$ & 0,953 \\
\hline "Concubinato" & 0,90 & $0,70-1,17$ & 0,424 \\
\hline Viúvo & 1,02 & $0,76-1,36$ & 0,909 \\
\hline \multicolumn{4}{|l|}{ Escolaridade ${ }^{\star \star}$} \\
\hline Analfabeto & 2,06 & $1,28-3,31$ & 0,003 \\
\hline 1a a $4 \underline{a}$ série do ensino fundamental & 2,03 & $1,30-3,16$ & 0,002 \\
\hline 5ạ a 8a série do ensino fundamental & 1,84 & $1,18-2,85$ & 0,007 \\
\hline 1ạ a 3ạ série do ensino médio & 1,25 & $0,82-1,91$ & 0,296 \\
\hline Superior incompleto ou completo & 1,00 & & \\
\hline \multicolumn{4}{|l|}{$\begin{array}{l}\text { Renda per capita } \\
\text { (salários mínimos) }^{\star \star}\end{array}$} \\
\hline$<0,5$ & 2,37 & $1,22-4,58$ & 0,011 \\
\hline $0,50-0,99$ & 2,51 & $1,39-4,54$ & 0,003 \\
\hline $1,00-1,99$ & 2,37 & $1,31-4,32$ & 0,005 \\
\hline $2,00-4,99$ & 1,73 & $0,96-3,11$ & 0,068 \\
\hline$\geq 5,00$ & 1,00 & & \\
\hline \multicolumn{4}{|l|}{ Cobertura PSF } \\
\hline Não & 1,00 & & \\
\hline Sim & 0,99 & $0,80-1,22$ & 0,928 \\
\hline
\end{tabular}

* Dados de sexo, idade, estado civil e cobertura PSF ajustados para todas as outras variáveis constantes nesta tabela; dados de escolaridade ajustados para sexo, idade, estado civil e cobertura PSF; dados de renda ajustados para sexo, idade, estado civil e cobertura PSF:

** Tendência linear com $p<0,01$.

ciar o meio e, conseqüentemente, dano à saúde psicológica 2,7,10.

Neste sentido, o fato da associação entre procedência e TMC perder significância quando ajustada por escolaridade é muito ilustrativo. Apesar de não existir uma direção clara na relação entre procedência e TMC, as discussões feitas sobre o tema incluem a recorrência 
do fator "exclusão social”. Composta por razões econômicas, que muitas vezes obrigam à migração e à exclusão do mercado formal de trabalho, este processo submete à integração num novo espaço social, com novos trabalhos e novas relações, que podem implicar incerteza extrema e tensão social, com repercussões permanentes ou temporárias sobre a saúde mental 11,19,21. Neste contexto, os resultados do presente estudo reafirmam maiores graus de escolaridade como fator de proteção.

Quanto à associação TMC e baixa renda per capita, também sinaliza diminuição de poder, incapacidade social e, portanto, maior susceptibilidade a doenças mentais 2,7,10. Na literatura o evento vital produtor de estresse "ter passado por dificuldades financeiras graves" é descrito como o mais fortemente associado ao TMC 17,20. No entanto, uma ressalva deve ser feita quanto a esta associação: ainda não está muito bem evidenciada qual é a verdadeira relação de causalidade entre pobreza, reações psicológicas e comportamento, sendo muito difícil, como já dito anteriormente, por intermédio de um estudo transversal como este, delinear tal direção 7,10,19.

Como se pode observar, não houve diferença significante entre as características sóciodemográficas das populações dos distritos analisados, assim como, evidenciou-se que a prevalência de TMC independe do tipo de cobertura assistencial, ou melhor, da presença ou ausência de cobertura pelo QUALIS. Esses resultados eram esperados devido à semelhança entre os distritos analisados, ambos muito carentes, classificados em 18o e 19o postos entre 31 subdistritos do Município de São Paulo 22. Não obstante, a ausência de diferença da prevalência de TMC entre as populações cobertas e não cobertas pelo PSF deve ser motivo de reflexão. Entre as possíveis causas para o achado de que a prevalência de TMC independe do tipo de cobertura assistencial estão a elevada vulnerabilidade dessas populações e a complexa rede causal dos TMC, que necessita de trabalho intersetorial e não somente do setor saúde. Há ainda a se considerar a inserção recente do programa na comunidade que impede a visualização de resultados mas não descarta a possibilidade de impacto a longo prazo. Também é necessário reconhecer a característica geral de abordagem dos problemas de saúde que tem o
PSF, ficando interrogada a possibilidade do programa em abordar especificamente esse problema de saúde, assim como de modificar sua evolução e/ou prognóstico. Outro aspecto a ser considerado são as características de assistência à saúde do Município de São Paulo, que oferece diversas alternativas, dentro e fora dos territórios analisados; inferências diretas sobre a presença ou ausência do PSF e sua relação com resultados expressos em indicadores de saúde nestas condições seriam inapropriadas. Finalmente, enfatizamos que neste estudo realizamos uma análise da prevalência de casos suspeitos de TMC, e não propriamente de casos diagnosticados, investigação que seria interessante em futuros estudos pois um quadro distinto pode ser revelado.

Esses resultados evidenciam a importância dos estudos epidemiológicos em saúde mental no Brasil, tanto no sentido de compreender melhor suas associações com as variáveis sócio-demográficas e, assim, orientar o reconhecimento de grupos de risco, quanto no sentido de se averiguar qual é a melhor forma de conduzir os casos que se enquadram nessa categoria. Neste último aspecto, vale a pena reforçar a questão do conhecimento dos TMC por parte dos médicos generalistas que, no exercício de sua profissão, devem estar conscientes dessas alterações e, conseqüentemente, de suas possíveis repercussões, por meio da adoção de um conceito biopsicossocial de saúde.

Além disso, revelam a real necessidade, apesar de implícita, da existência e disponibilidade de uma abordagem voltada, especialmente, para saúde mental na esfera do atendimento primário, principalmente, em áreas relacionadas às variáveis sócio-demográficas associadas, neste presente trabalho, aos TMC.

Durante a elaboração deste projeto e, em seguida, com a obtenção dos resultados e com o estudo mais aprofundado sobre o tema na literatura, muitas outras dúvidas surgiram sobre a questão dos TMC e os serviços de saúde. Como sugestões para futuros estudos nessa área, ressalta-se a importância em se investigar quais são os fatores prognósticos de maior valor em relação aos TMC, qual a perspectiva, a eficácia e a viabilidade em tratá-los e, mais especificamente, em relação ao PSF, se tal abordagem é feita, como ela é feita e qual a capacidade desse programa em trabalhar também com essa área. 


\section{Resumo}

Vários países apresentam crescimento da prevalência dos transtornos mentais comuns (TMC). Poucos casos são diagnosticados e tratados adequadamente porque os serviços tradicionais de saúde raramente estão preparados para lidar com esse problema. O Programa Saúde da Família (PSF), implementado no Brasil desde 1995/1996, representa um novo modelo de atenção com potencial para melhor atuação nesses casos. Este estudo objetiva investigar a prevalência de TMC segundo a cobertura PSF e certos fatores de risco sóciodemográficos. Um inquérito de saúde e acesso a serviços foi realizado de janeiro a março de 2001 em áreas periféricas do Município de São Paulo, parcialmente cobertas pelo PSF, e incluiu o rastreamento de TMC em 2.337 indivíduos maiores de 15 anos de idade. Não se observou diferença significante na prevalência de TMC segundo a cobertura PSF. A prevalência foi significantemente maior nas mulheres $(R P=1,34)$, idosos $(R P=1,56)$ e nas categorias de menor renda $(R P=2,64)$ ou de menor escolaridade $(R P=2,83)$. Os TMC se mostraram associados a indicadores de desvantagem social, implicando a necessidade de focalização do problema e dos grupos de risco específicos para maior impacto da atenção.

Transtornos Mentais; Programa Saúde da Família; Avaliação dos Serviços

\section{Colaboradores}

L. Maragno e R. J. Gianini realizaram a análise dos dados e a redação final. M. Goldbaum foi responsável pela concepção do projeto, trabalho de campo, análise dos dados e redação final. H. M. D. Novaes foi responsável pela concepção do projeto e redação final. C. L. G. César participou da concepção do projeto e do trabalho de campo.

\section{Agradecimentos}

À Dra. Maria Cecília Goi Porto Alves, da Superintendência de Controle de Endemias (SUCEN), pela assessoria no processo amostral. Financiamento: Projeto FAPESP no 1998/15246-3; Iniciação Científica, FAPESP, processo no 02/09947-6; Bolsa CNPq processos 303617/85-3 e 522321/96-8; e Projeto DST/AIDS/MS processo 635/99.

\section{Referências}

1. Menezes PR. Princípios de epidemiologia psiquiátrica. In: Almeida OP, Dratcu L, Laranjeira R, organizadores. Manual de psiquiatria. Rio de Janeiro: Guanabara Koogan; 1996. p. 43-55.

2. Coutinho ESF, Almeida Filho N, Mari JJ. Fatores de risco para morbidade psiquiátrica menor: resultados de um estudo transversal em três áreas urbanas no Brasil. Rev Psiq Clín 1999; 26(5). http:/ / www.hcnet.usp.br/ipq/revista (acessado em 25/ Jun/2002).

3. Jenkins R, Lewis G, Bebbington P, Brugha T, Farrell M, Gill B, et al. The National Psychiatric Morbidity Surveys of Great Britain - initial findings from the household survey. Psycho Med 1997; 27:775-89.

4. Almeida-Filho N, Mari JJ, Coutinho E, Franca JF, Fernandes J, Andreoli SB, et al. Brazilian multicentric study of psychiatric morbidity. Methodological features and prevalence estimates. Br J Psychiatry 1997; 171:524-9.

5. Andrade LHSG, Lólio CA, Gentil V, Laurenti R. Epidemiologia dos transtornos mentais em uma área definida de captação da Cidade de São Paulo, Brasil. Rev Psiq Clín 1999; 26(5). http://www. hcnet.usp.br/ipq/revista (acessado em 25/Jun/ 2002).

6. Santos MESB. Transtornos mentais comuns em pacientes com AIDS que fazem uso de anti-retrovirais no Estado de São Paulo, Brasil [Dissertação de Mestrado]. São Paulo: Faculdade de Medicina, Universidade de São Paulo; 2002.

7. Ludermir AB, Melo Filho DA. Condições de vida e estrutura ocupacional associadas a transtornos mentais comuns. Rev Saúde Pública 2002; 36:21321.

8. Lima MS, Soares BGO, Mari JJ. Saúde e doença mental em Pelotas, RS: dados de um estudo populacional. Rev Psiq Clín 1999; 26(5). http://www. hcnet.usp.br/ipq/revista (acessado em 25/Jun/ 2002).

9. Bhagwanjee A, Parekh A, Paruk Z, Petersen I, Subedar H. Prevalence of minor psychiatric disorders in an adult African rural community in South Africa. Psychol Med 1998; 28:1137-47.

10. Patel V, Araya R, Lima M, Ludermir A, Todd C. Women, poverty and common mental disorders in four restructuring societies. Soc Sci Med 1999; 49:1461-71.

11. Souza MFM, Silva GR. Risco de distúrbios psiquiátricos menores em área metropolitana na região sudeste do Brasil. Rev Saúde Pública 1998; 32:50-8.

12. Marques RM, Mendes A. Atenção básica e Programa Saúde da Família (PSF): novos rumos para a política de saúde e seu financiamento. Ciênc Saúde Coletiva 2003; 8:403-15.

13. Viana AL, Dal Poz MR. A reforma do sistema de saúde no Brasil e o Programa Saúde da Família. Physis 1998; 8:11-48.

14. Conill EM. Políticas de atenção primária e reformas sanitárias: discutindo a avaliação a partir da análise do Programa Saúde da Família em Florianópolis, Santa Catarina, Brasil, 1994-2000. Cad Saúde Pública 2002; 18 Suppl:191-202. 
15. Santana ML, Carmagnani MI. Programa de Saúde da Família no Brasil: um enfoque sobre os pressupostos básicos, operacionalização e vantagens. Saúde Soc 2001; 10:33-54.

16. Mari JJ, Willians P. A validity study of a psychiatric screening questionnaire (SRQ-20) in primary care in the city of São Paulo. Br J Psychiatry 1996; 148:23-6.

17. Costa AG, Ludermir AB. Transtornos mentais comuns e apoio social: estudo em comunidade rural da Zona da Mata de Pernambuco, Brasil. Cad Saúde Pública 2005; 21:73-9.

18. Weich S, Lewis G. Poverty, unemployment, and common mental disorders: population based cohort study. BMJ 1998; 317:115-9.

19. Mari JJ. Psychiatric morbidity in three primary medical care clinics in the city of São Paulo. Soc Psychiatry 1987; 22:129-38.
20. Lopes SC, Faerstein E, Chor D. Eventos de vida produtores de estresse e transtornos mentais comuns: resultados do Estudo Pró-Saúde. Cad Saúde Pública 2003; 19:1713-20.

21. Almeida-Filho N, Mari JJ, Coutinho ESF. Migração, inserção produtiva e saúde mental na modernidade tardia: novas evidências do estudo multicêntrico de morbidade psiquiátrica em áreas metropolitanas brasileiras. Rev Psiq Clin 1999; 26(5). http://www.hcnet.usp.br/ipq/revista (acessado em 25/Jun/2002).

22. Pedroso M. Desenvolvimento humano no $\mathrm{Mu}$ nicípio de São Paulo [Dissertação de Mestrado]. São Paulo: Pontifícia Universidade Católica de São Paulo; 2003.

Recebido em 02/Mai/2005

Versão final reapresentada em 03/Nov/2005

Aprovado em 14/Dez/2005 